OPEN ACCESS

Edited by: Norberto Perico, Istituto di Ricerche Farmacologiche Mario Negri, Italy

Reviewed by:

Miriam Zacchia, University of Campania Luigi Vanvitelli,

Domenino Santoro, University of Messina, Italy

*Correspondence:

Miaomiao Lu miaomiaolu2012@163.com

Specialty section: This article was submitted to Renal Pharmacology, a section of the journal Frontiers in Pharmacology

Received: 17 December 2020 Accepted: 15 April 2021 Published: 29 April 2021

Citation:

Liu W, Li L, Zhang X, Dong H and Lu M (2021) Efficacy and Safety of Veverimer in the Treatment of Metabolic Acidosis Caused by Chronic Kidney Disease: A Meta-analysis.

Front. Pharmacol. 12:643128. doi: 10.3389/fphar.2021.643128

\section{Efficacy and Safety of Veverimer in the Treatment of Metabolic Acidosis Caused by Chronic Kidney Disease: A Meta-analysis}

\author{
Wenlin Liu, Lili Li, Xuemei Zhang, Haonan Dong and Miaomiao Lu* \\ Department of Nephrology, The First Affiliated Hospital of Jinzhou Medical University, Jinzhou, China
}

Metabolic acidosis is a common complication of chronic kidney disease (CKD). Veverimer is an orally administrated, free amine polymer with high capacity and binding selectivity to hydrochloric acid from the gastrointestinal tract. This study pooled the current evidence of the efficacy and safety of veverimer for the treatment of metabolic acidosis associated with CKD. We conducted a systematic literature search on PubMed, Embase, and Cochrane Central for relevant randomized controlled trials (RCTs) in June 2020. In this study, three RCTs with 548 patients were included in our analysis. The analysis revealed that veverimer was associated with increased bicarbonate level of patients (weight mean difference [WMD] 3.08, 95\% confidence interval [CI] [2.40, 3.77], $p<0.001$ ) and improved physical function compared with placebo measured by Kidney Disease and Quality of Life Short Form 36, question 3 (physical functioning domain) (KDQoL-PFD) score (WMD 5.25, 95\% Cl [1.58, 8.92], $p=0.005)$. For safety outcomes, both groups exhibited similar risks for developing headache, diarrhea, flatulence, and hyperkalemia. In conclusion, current clinical evidence indicates that veverimer is efficacious and safe against metabolic acidosis related to CKD compared with placebo. Further research comparing long-term veverimer use with traditional alkali therapy is needed.

Keywords: metabolic acidosis, chronic kidney disease, serum bicarbonate, veverimer, meta-analysis

\section{INTRODUCTION}

Chronic kidney disease (CKD) is a long-term structural or functional disorder of the kidneys, manifested by elevated serum levels of creatinine, cystatin C, or blood urea nitrogen (Drawz and Rahman, 2015). The estimated prevalence rate for all stages of CKD was 13\% (Hill et al., 2016). Most patients with CKD are at risk for accelerated cardiovascular disease and death, with significantly impaired life expectancy and quality of life.

Metabolic acidosis is a common and persistent complication of $\mathrm{CKD}$, which contributes to the continued progression of CKD. It is associated with a decrease in total renal ammonium excretion, titratable acid excretion, and bicarbonate reabsorption as a result of a decline in glomerular filtration rate (GFR) (Kraut and Madias, 2016). Previous studies have shown that chronic disturbances in serum acid excretion with increased serum bicarbonate are related to increased risk for renal disease progression, heart failure, and allcause mortality (Shah et al., 2009; Menon et al., 2010; Raphael et al., 2011; Dobre et al., 2013).

Traditional treatments of metabolic acidosis mainly involve oral alkali supplementation. However, previous evidence demonstrated that oral alkali supplementation might result in edema, atherosclerosis progression (Lomashvili et al., 2006). In addition, the management of dietary interventions was proved difficult to fulfill, resulting in suboptimal treatment outcomes. 
Veverimer (TRC101) is a novel oral and nonabsorbable hydrochloric acid adhesive. It is not an ion exchanger and does not introduce sodium ions. The structure and nature of veverimer dictate that it is protonated upon ingestion and selectively binds to anions, resulting in a reduction and removal of hydrochloric acid from the gastrointestinal tract (Wesson et al., 2019b; Adrogué and Madias, 2020). Data obtained from clinical trials (Bushinsky et al., 2018; Wesson et al., 2019a; Wesson et al., 2019b) have revealed that veverimer was effective against acidosis related to CKD, thereby improving patients' bicarbonate level. The endogenous increase in serum bicarbonate without sodium retention is a unique feature of veverimer. However, some researchers have found that the mechanism of veverimer parallels the persistent vomiting to remove gastric acid. The difference is that add bicarbonate level of persistent vomiting is accompanied by the loss of endogenous fluid and ions; whereas the increased endogenous bicarbonate with veverimer does not lose fluid, sodium and potassium, etc. (Adrogué and Madias, 2020). No systematic approach has been conducted to evaluate the safety and efficacy of veverimer for the treatment of metabolic acidosis associated with CKD. Therefore, we conducted a meta-analysis to analyze the efficacy and safety veverimer.

\section{METHODS}

\section{Search Strategy}

We conducted a systematic search for relevant studies evaluating the use of veverimer for the treatment of acidosis in patients with CKD. This meta-analysis protocol was performed in strict accordance with the preferred reporting items for systematic reviews and metaanalysis (PRISMA) statement (Moher et al., 2009). MEDLINE (via PubMed), Embase, and the Cochrane Central Register of Controlled Trials were searched from 2000 to June 2020. The search was conducted using Medical Subject Headings (MeSH) terms and the keywords "veverimer," "CKD," "chronic renal disease," "chronic kidney disease," "kidney failure, chronic," and "metabolic acidosis." In addition, the relevant trials were accessed using the ClinicalTrials.gov platform. Related references were screened to avoid omission. There were no language restrictions.

\section{Eligibility Criteria}

Inclusion criteria: 1) Randomized controlled trials (RCTs); 2) Studies evaluating veverimer use in adult subjects ( $\geq 18$ years) with acidosis and CKD. Metabolic acidosis refers acid-base disturbance, which caused by increased $\mathrm{H}^{+}$or loss of $\mathrm{HCO}^{-}$ in extracellular fluid $\left\{\left[\mathrm{HCO}^{-}\right]<22 \mathrm{mmol} / \mathrm{L}\right\}$ (Raphael, 2019; Gone and Chen, 2020). CKD is defined as a progressive disease of the kidneys with structural and functional disorders or GFR lower than $60 \mathrm{ml} /\left(\min \cdot 1.73 \mathrm{~m}^{2}\right)$ for at least 3 months (Peng et al., 2019), patients of chronic renal insufficiency often leads to high anion gap (AG) acidoses; 3) Patients randomly assigned to experimental and control group. Exclusion criteria: reviews, studies with insufficient data, and patients with normal serum bicarbonate were excluded from the analysis. All eligible studies had a comparison of veverimer with the placebo group.

\section{Quality Assessment}

Two authors independently used in accordance with the Cochran Collaboration risk of bias tool for evaluating the quality of RCTs as described in a previous study (Higgins et al., 2011), following selection bias, performance bias, detection bias, attrition bias, reporting bias were assessed. In brief, these items were rated as "low risk", "high risk", or "unclear" evaluating individual study. Discrepancies regarding the quality assessment were solved by discussion with another experienced investigator.

\section{Extraction of Data and Outcome}

Two review authors (WL and LL) extracted data from these studies, including the following information: 1) first author, 2) number of subjects, 3) country, 4) recruitment period, 5) study design, 6) mean age of patients, 7) sex, 8) primary outcomes, 9) follow-up time (10) inclusion criteria, and 11) national clinical trial number. In addition, we summarized the baseline characteristics of the study population. Any disagreements regarding the inclusion or exclusion of the articles were resolved by consensus or adjudicated by the third reviewer (ML).

For the efficacy outcome, changes in bicarbonate concentration over time were the primary endpoint. The secondary efficacy endpoints include Kidney Disease and Quality of Life Short Form (SF) 36, question 3 (physical functioning domain) (KDQoL-PFD) score, and repeated chair stand (s). In addition, we evaluated the adverse events of veverimer in patients, which include diarrhea, headache, flatulence, hyperkalemia, the decreased level of GFR and influenza.

\section{Statistical Analysis}

Review Manager (version 5.3) was used for all data analyses in this study. Enumeration data were used to analyze the risk ratio and measurement data to obtain the weighted mean differences (WMDs) to compare the endpoints of the two groups. For each effect size, we provide values and 95\% confidence intervals (CIs). $p<0.05$ was considered statistically significant. Besides, $\mathrm{I}^{2}$ index was performed to evaluate heterogeneity. $\mathrm{I}^{2}>50 \%$ and $p<0.1$ were considered to indicate significant heterogeneity, and the randomeffect model was selected, while $\mathrm{I}^{2}<50 \%$ and $p>0.1$ represent good homogeneity, and the fixed-effect model was chosen for analysis. Therefore, the meta-analysis was performed using the fixed-effect model with the DerSimonian and Laird method.

\section{RESULTS}

\section{Literature Search Results}

The search and screen process was illustrated in Figure 1. Literature search to 1,189 studies. After excluding 74 duplicate studies and 984 non-RCT studies, 131 articles remained. Of these 131 studies, three double-blinded RCTs (involving 135 patients randomly assigned to the phase $1 / 2$ study, 217 patients randomly rationed to the 12 week-clinical trail, and 114 patients and 82 patients to veverimer $v s$. placebo in extension phase, respectively) (Bushinsky et al., 2018; Wesson et al., 2019a; Wesson et al., $2019 b$ ) were included in the quantitative analysis. 


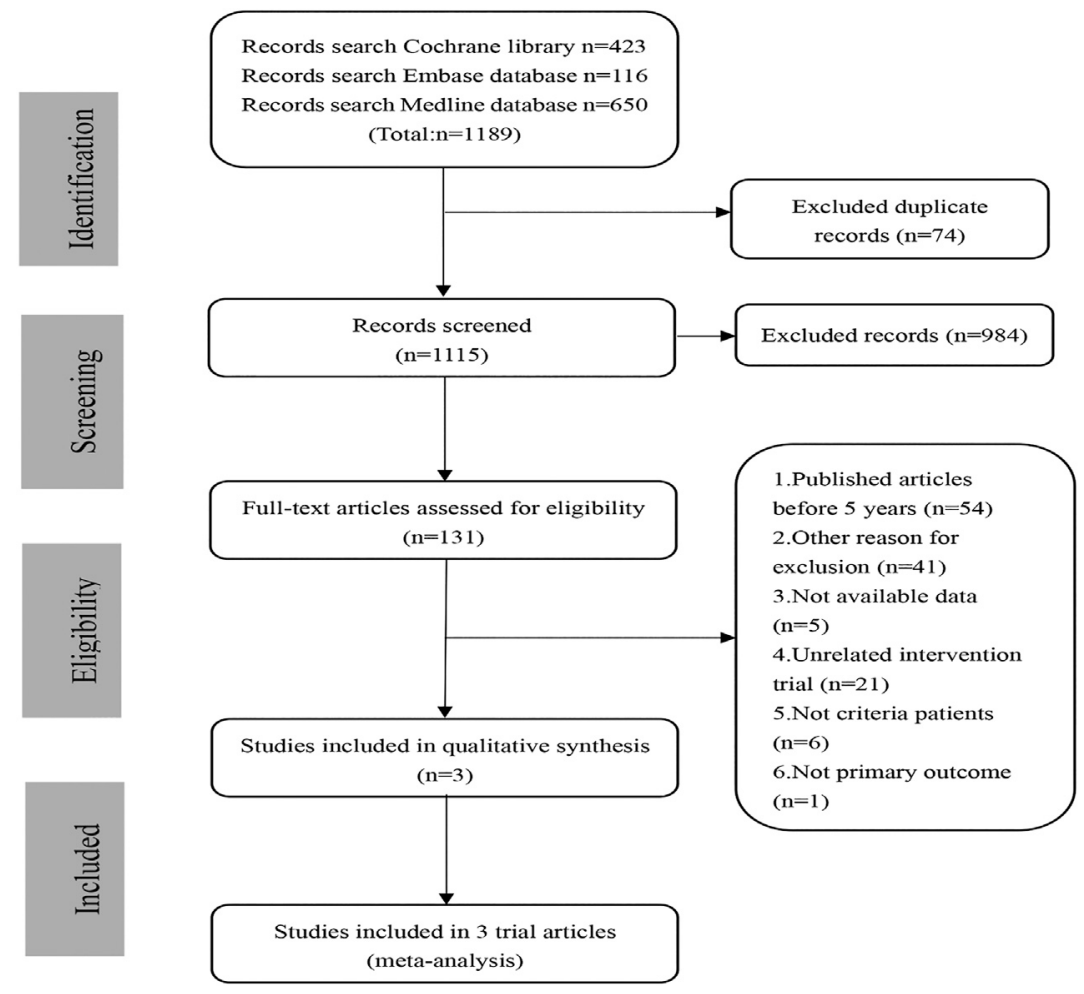

FIGURE 1 | Flowchart for data extraction.

\section{Basic Characteristics and Quality Assessment of Studies}

The baseline characteristics of the included studies are presented in Table 1. Notably, the 548 participants in the three trials were patients with acidosis, with 342 in the treatment group and 206 in the control group. The population of criteria for initiation was $12-20 \mathrm{mmol} / \mathrm{L}$ for $\left[\mathrm{HCO}^{-}\right]$at hospitals and specialty clinics in Bulgaria, Georgia and other countries. Participants were treated with veverimer-placebo for 2, 12 and 52 weeks. Available data about changing from baseline in bicarbonate, physical function and safety assessment were obtained. The baseline characteristics of the studies are presented in Table 2. All of the studies were at low risk of bias (Figure 2).

\section{The Efficacy of Veverimer Compared With Placebo}

Twenty patients were excluded from the primary outcome because of missing basic bicarbonate data. Among the remaining 528 subjects, the use of veverimer was associated with significantly increased serum bicarbonate level compared with the control group (WMD 3.08, 95\% CI $[2.40,3.77], p<0.001)$, and the heterogeneity was low $\left(\mathrm{I}^{2}=15 \%\right.$, $p>0.05$ ) (Figure 3). Regarding the subgroup indicators affecting the primary outcome, Wesson et al. (2019b) found that there was a significant improvement in $\left[\mathrm{HCO}^{-}\right]$on average in the age group, sex, baseline bicarbonate, and screening eGFR groups of patients who took veverimer treatment, however this performance was not present in receiving acid reducing drug or alkali therapy patients. Figure 4 illustrates the change in bicarbonate concentration between the groups during the 52 weeks. These data also indicated that veverimer use was associated with persistent correction of bicarbonate level compared with the placebo group. In addition to the primary endpoint, pooled analysis of two studies (Wesson et al., 2019a; Wesson et al., 2019b) involving 381 participants revealed that veverimer significantly improved KDQoL-PFD score compared with placebo (WMD 5.25, $95 \% \mathrm{CI}[1.58,8.92], p<0.01)$ with low heterogeneity $\left(\mathrm{I}^{2}=0, \mathrm{Z}=2.81\right.$, $p=0.97$ ) (Figure 5A). For repeated chair stand test time, after pooling data from two trials (Wesson et al., 2019a; Wesson et al., 2019b) involving 380 participants, veverimer was not associated with improved repeated chair stand(s) (WMD 0.84, 95\% CI $[-0.73$, $2.40], p=0.29)$ with low heterogeneity $\left(\mathrm{I}^{2}=0, p=0.95\right)$ (Figure $5 \mathrm{~B}$ ).

\section{The Safety Outcomes of Veverimer Compared With Placebo}

Headache, diarrhea, flatulence, and hyperkalemia were considered the safety endpoints in our meta-analysis. In the three studies, veverimer was not associated with increased risk for developing treatmentemergent headache and diarrhea compared with the placebo group (diarrhea: RR 1.71, 95\% CI [0.86, 3.40], $p=0.13, \mathrm{I}^{2}=0$; headache: 0.79, $95 \%$ CI $[0.44,1.41], p=0.42, \mathrm{I}^{2}=29 \%$ ) (Figure 6). Also, there are two studies (Wesson et al., 2019a; Wesson et al., 2019b) reporting the risk of flatulence and hyperkalemia, but no difference between the two groups was observed (flatulence: RR 1.75, 95\% CI [0.88, 3.48], $p=0.11$, $\mathrm{I}^{2}=0$; hyperkalemia: RR $1.48,95 \%$ CI $[0.77,2.86], p=0.24, \mathrm{I}^{2}=0$ ). Besides, GFR decreased and influenza also were discovered in some 
TABLE 1 | Characteristics of included randomized clinical trials.

\begin{tabular}{|c|c|c|c|c|c|c|c|c|c|c|}
\hline References & $\begin{array}{c}\text { Patients, } \\
n\end{array}$ & Country & $\begin{array}{c}\text { Recruitment } \\
\text { period }\end{array}$ & $\begin{array}{c}\text { Design } \\
\text { and setting }\end{array}$ & $\begin{array}{l}\text { Mean } \\
\text { age of } \\
\text { patients, } \\
\text { years } \\
\text { (SD) }\end{array}$ & $\begin{array}{c}\text { Sex } \\
\text { (male, } \\
\text { female) }\end{array}$ & $\begin{array}{l}\text { Primary } \\
\text { outcome }\end{array}$ & $\begin{array}{c}\text { Duration } \\
\text { of follow- } \\
\text { up } \\
\text { (week) }\end{array}$ & $\begin{array}{c}\text { Criteria } \\
\text { for veverimer- } \\
\text { placebo } \\
\text { initiation }\end{array}$ & $\begin{array}{c}\text { No of } \\
\text { subjects }\end{array}$ \\
\hline $\begin{array}{l}\text { Wesson et } \\
\text { al, 2019-06 }\end{array}$ & 196 & $\begin{array}{l}\text { Bulgaria, } \\
\text { Georgia, } \\
\text { Hungary, } \\
\text { Serbia, } \\
\text { Slovenia, } \\
\text { Ukraine, } \\
\text { USA }\end{array}$ & $2017-18$ & $\begin{array}{l}\text { Multicentre, } \\
\text { CKD and } \\
\text { metabolic } \\
\text { acidosis } \\
\text { patients }\end{array}$ & $\begin{array}{l}\text { Veverimer } \\
62.9(12.1) ; \\
\text { Placebo } \\
61.7 \text { (11.9) }\end{array}$ & $\begin{array}{l}61 \% \\
39 \%\end{array}$ & $\begin{array}{l}\text { Long-term } \\
\text { safety }\end{array}$ & 40 & $\begin{array}{l}\text { eGFR } \\
20-40 \mathrm{ml} / \mathrm{min} \\
\text { per } 1.73 \mathrm{~m}^{2} \text {, } \\
\text { serum } \\
\text { bicarbonate } \\
12-20 \mathrm{mmol} / \mathrm{L}\end{array}$ & NCT03390842 \\
\hline $\begin{array}{l}\text { Wesson et } \\
\text { al, 2019-03 }\end{array}$ & 217 & $\begin{array}{l}\text { Bulgaria, } \\
\text { Georgia, } \\
\text { Hungary, } \\
\text { Serbia, } \\
\text { Slovenia, } \\
\text { Ukraine, } \\
\text { USA }\end{array}$ & $2017-18$ & $\begin{array}{l}\text { Multicentre, } \\
\text { CKD and } \\
\text { metabolic } \\
\text { acidosis } \\
\text { patients }\end{array}$ & $\begin{array}{l}\text { Veverimer } \\
62.9(12.6) ; \\
\text { Placebo } \\
63.2(12.1)\end{array}$ & $\begin{array}{l}62.5 \% \\
37.5 \%\end{array}$ & $\begin{array}{l}\text { Change from } \\
\text { baseline in } \\
\text { blood } \\
\text { bicarbonate }\end{array}$ & 12 & $\begin{array}{l}\text { eGFR } \\
20-40 \mathrm{ml} / \mathrm{min} \\
\text { per } 1.73 \mathrm{~m}^{2} \text {, } \\
\text { serum } \\
\text { bicarbonate } \\
12-20 \mathrm{mmol} / \mathrm{L}\end{array}$ & NCT03317444 \\
\hline $\begin{array}{l}\text { Bushinsky et } \\
\text { al, } 2018\end{array}$ & 135 & $\begin{array}{l}\text { Bulgaria, } \\
\text { Georgia }\end{array}$ & 2016-20 & $\begin{array}{l}\text { Stage } 3 \text { or } 4 \\
\text { CKD and } \\
\text { metabolic } \\
\text { acidosis } \\
\text { patients }\end{array}$ & $\begin{array}{l}\text { Veverimer } \\
60(13) ; \\
\text { Placebo } \\
61(12)\end{array}$ & $\begin{array}{c}64 \% \\
36 \%\end{array}$ & $\begin{array}{l}\text { Change from } \\
\text { baseline to the } \\
\text { end of } \\
\text { treatment (Day } \\
\text { 15) in serum } \\
\text { bicarbonate } \\
\text { within each } \\
\text { individual } \\
\text { TRC101 dose } \\
\text { group }\end{array}$ & 2 & $\begin{array}{l}\text { Patients had a } \\
\text { mean baseline } \\
\text { eGFR of } 35 \mathrm{ml} / \\
\text { min per } \\
1.73 \mathrm{~m}^{2} \text {, a mean } \\
\text { baseline serum } \\
\text { bicarbonate of } \\
17.7 \mathrm{mmol} / \mathrm{L}\end{array}$ & NCT02809183 \\
\hline
\end{tabular}

TABLE 2 | Baseline characteristics of patients in the meta-analysis trials.

Veverimer $(n=342)$

Age, years

Sex

Male

Female

Hypertension

Left ventricular hypertrophy

Serum bicarbonate $(\mathrm{mmol} / \mathrm{L})$

$>18 \mathrm{mmol} / \mathrm{L}$

$\leq 18 \mathrm{mmol} / \mathrm{L}$

Diabetes mellitus

Estimated GFR (ml/min per $\left.1.73 \mathrm{~m}^{2}\right)$

KDQOL SF-36 physical function domain total score

Repeated chair stand (s)
$61.9(12.6)$
209/342 (61\%)
$133 / 342$ (39\%)
$326 / 342$ (95\%)
145/342 (42\%)
17.4 (1.3)
$80 / 238(34 \%)$
$158 / 238(66 \%)$
220/342 (64\%)
$31.2(8.6)$
53.0 (23.0)
19.5 (14.3)

Placebo $(n=206)$

$61.9(12.0)$

130/206 (63\%)

$76 / 206(37 \%)$

199/206 (97\%)

$81 / 206$ (39\%)

$17.3(1.5)$

$55 / 175$ (31\%)

$120 / 175(69 \%)$

142/206 (69\%)

$29.9(8.3)$

$54.9(26.7)$

18.3 (13.0)

Data are mean (SD) or $\mathrm{n}(\%)$.

a Veverimer $\mathrm{n}=237$; placebo $\mathrm{n}=175$.

${ }^{b}$ Veverimer $n=225$; placebo $n=160$.

cases, but they did not have an effect on safety outcome (Bushinsky et al., 2018; Wesson et al., 2019a). Notably, both groups had similar endpoints of adverse events $(p>0.05)$ (Figure 6).

\section{DISCUSSION}

Our study pooled 548 individuals with acidosis associated with CKD from three high quality RCTs. The analysis demonstrated that veverimer use was effective against acidosis in patients with CKD, with a mean increase in serum bicarbonate level of by an average of $3.08 \mathrm{mmol} / \mathrm{L}$ compared with placebo, and was related to improved physical function. Besides, the pooled results indicated that veverimer use was not associated with an increased risk of developing adverse events. Heterogeneity analysis of all the included outcomes was low, indicating a high level of clinical evidence. The observed outcomes strongly support the use of veverimer is beneficial for the treatment of acidosis related to $\mathrm{CKD}$. 


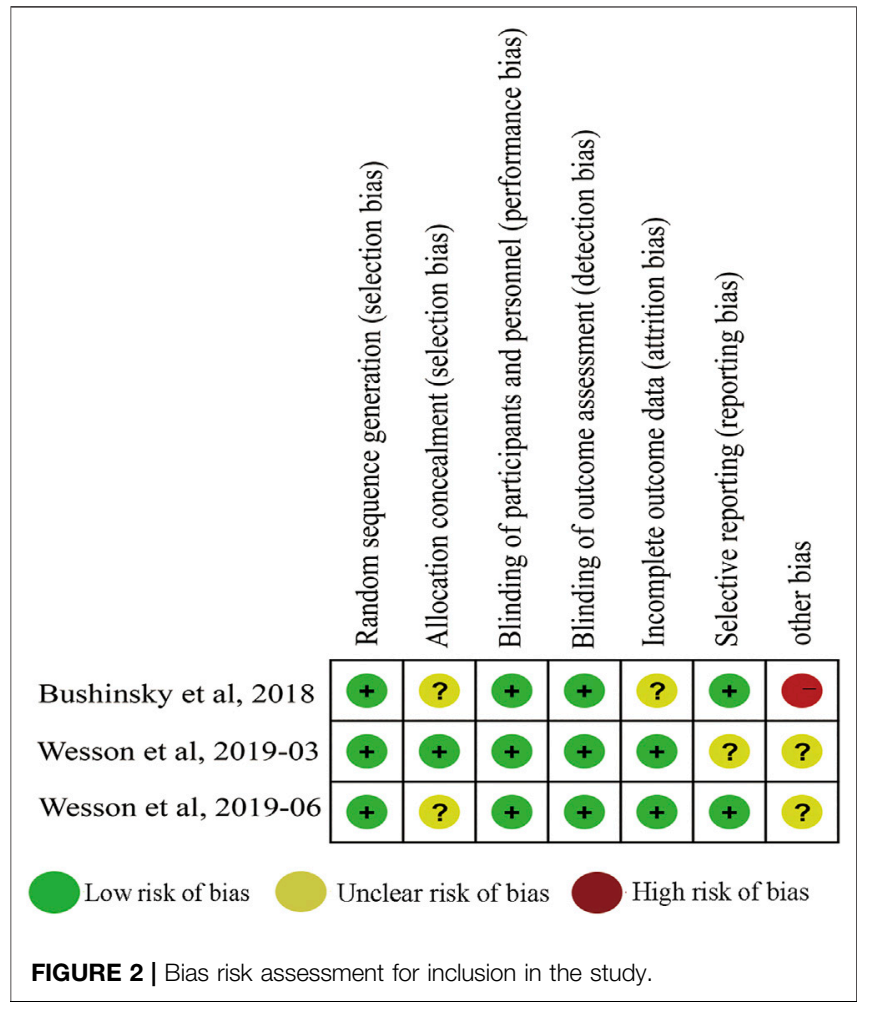

value of serum bicarbonate concentration to $\geq 22 \mathrm{mmol} / \mathrm{L}$ (Bailie and Massry, 2005). First-line therapy of metabolic acidosis includes reducing acidic foods intakes or using alkalization treatments that maintain total serum carbon dioxide (Susantitaphong et al., 2012). In available data from 20 patients with metabolic acidosis, Matthew K. et al. found that for every $0.1 \mathrm{mmol} / \mathrm{kg}$ increase in the daily dose of oral sodium bicarbonate treatment during two week periods, serum bicarbonate increased by $0.33 \mathrm{mmol} / \mathrm{L}$ (Abramowitz et al., 2013). Besides, Lambers et al. showed the dietary intervention also reduced the renal acid load of the patients to varying degrees (Lambers Heerspink et al., 2012). However, studies revealed that sodium bicarbonate therapy had been found to introduce additional sodium into the body, which may increase the potential risk of sodium-sensitive comorbidities such as edema and hypertension, making it necessary for patients to take diuretics and antihypertensives to mitigate the inevitable complications. Furthermore, previous studies revealed an undesirable effect of alkalinization therapy on cardiovascular events (Kendrick et al., 2018; Raphael, 2019; Klaerner et al., 2020). Moreover, alkalization treatments may reduce the effect of nephroprotective agents, indicating that traditional treatments may aggravate the deterioration of renal function (Lambers Heerspink et al., 2012; Chen and Abramowitz, 2019; Navaneethan et al., 2019).

Veverimer, as a hydrochloric acid adhesive, can effectively combine with and remove hydrochloric acid from the

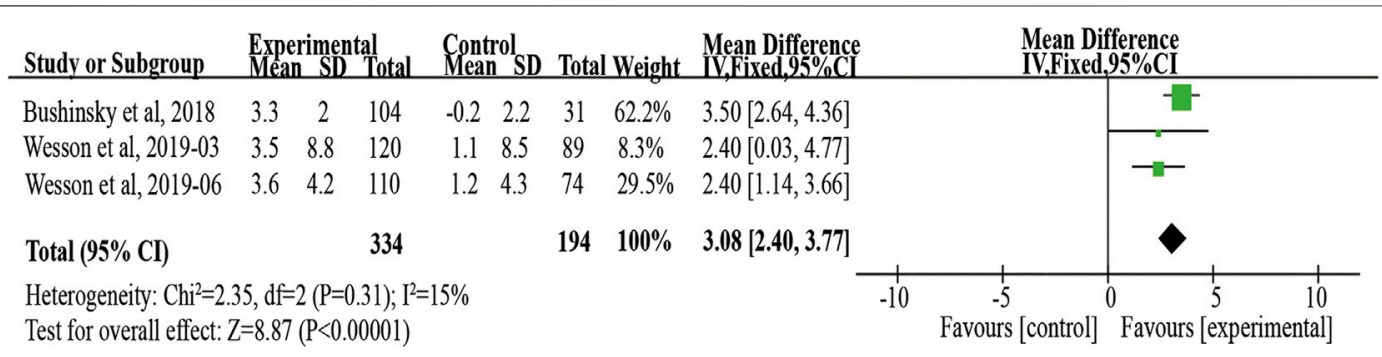

FIGURE 3 | Forest plot for change in serum bicarbonate. IV, independent variable method; $95 \% \mathrm{Cl}, 95 \%$ confidence interval.

Patients with acidosis usually have no obvious clinical symptoms and are aware of their acid-base disorder through a chemistry panel. In the chronic renal insufficiency cohort study, it was shown that patients with less than $22 \mathrm{mmol} / \mathrm{L}$ had an almost 2 -fold increased risk of CKD progression (Dobre et al., 2015). Notably, it is well known that eGFR is a critical diagnostic criterion for the diagnosis of CKD (Goldenstein et al., 2014). Extensive research has provided clinical evidence that patients with CKD with impaired GFR are likely to develop metabolic acidosis. Moreover, studies have revealed that an increase in bicarbonate concentration to the normal range can delay renal replacement therapy in patients with CKD and slow down the decline in the eGFR (Di Iorio et al., 2019).

Since metabolic acidosis can lead to low function of multiple organs in the body, we need to focus on the treatment of acidosis. The National Kidney Foundation guidelines recommend maintaining the gastrointestinal tract, thereby increasing the concentration of bicarbonate without causing hypervolemia and fluid loss. Therefore, chloride removal by veverimer may be more suitable for CKD patients with various organic diseases. KDQoL-PFD is a widely used questionnaire based on the quality of daily life and the physical function of patients with kidney disease. It was demonstrated that both alkalization therapy and veverimer could improve KDQoL-PFD (Abramowitz et al., 2013; Witham et al., 2015). According to the report, the clinical difference of KDQoL subscales is at least 3-5 points before and after treatment (Samsa et al., 1999; Clement et al., 2009; Collister et al., 2016). In our pooled analysis, the average change in the veverimer group was 6.4 points, and that in the placebo group was only 1.1 points, with a significant intergroup difference of 5.25 (95\% CI [1.58, 8.92], $p<0.01$ ).

Nevertheless, the treatment group improved by $1.8 \mathrm{~s}$ on average in the repeated chair stand test, which was also used to assess physical 


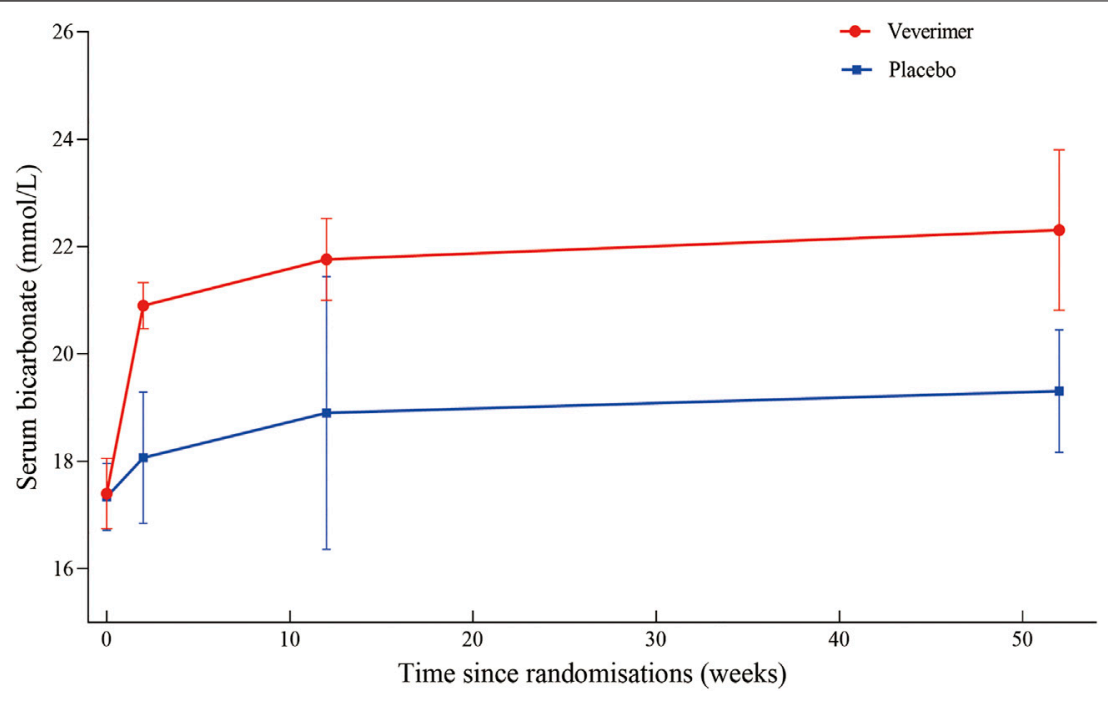

FIGURE 4 | Changes in bicarbonate levels in the two groups during the 52 weeks.

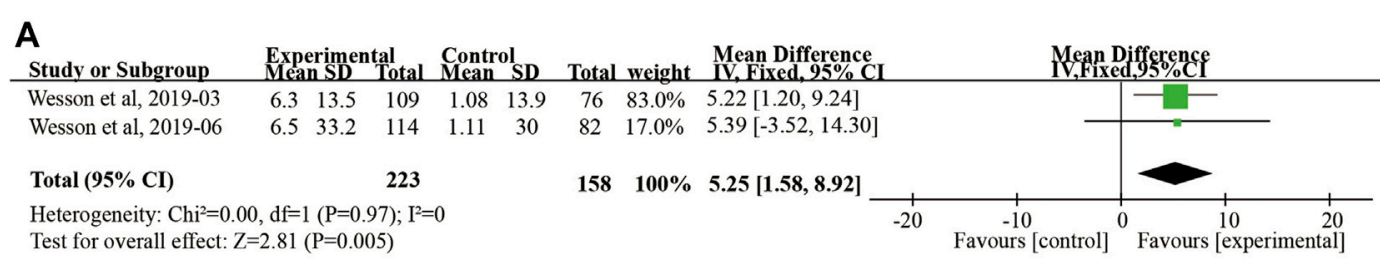

\section{KDQoL-PFD score}

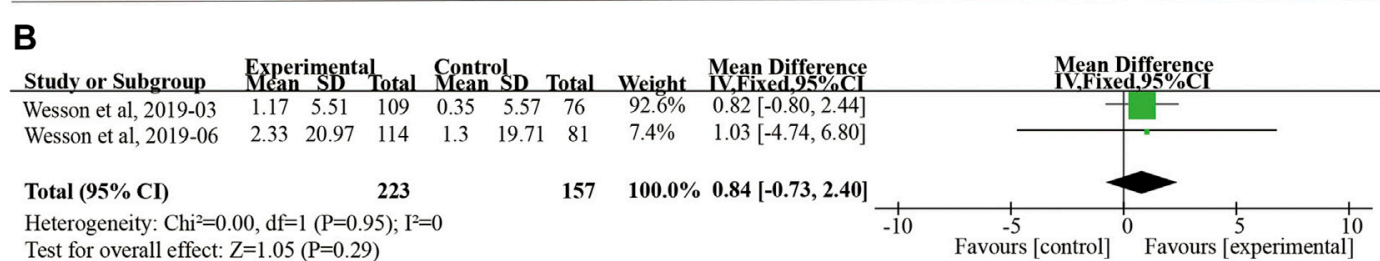

Repeated chair stand(s)

FIGURE 5 | Forest plot for change in physical function. (A) Difference in KDQoL-PFD score among studies included in the meta-analysis. (B) Difference in repeated chair stand (s) among studies included in the meta-analysis. IV, independent variable method; 95\% Cl, 95\% confidence interval.

function. This test score was similar to the minimum clinical difference value, indicating that veverimer cannot significantly extend the time of repeating chair stand test (WMD 0.84, 95\% CI [-0.73, 2.40], $p=0.29$ ). Based on the current clinical score, we speculate that this finding may be related to the sample size and the fact that the repeated chair stand test was in only two trials, thereby limiting the judgment regarding the effect of veverimer treatment on physical function. Notably, because of the lack of trials comparing alkalization and veverimer interventions, further clinical trials are needed to evaluate the clinical advantage of veverimer over traditional treatments.
We also further analyzed safety outcomes. Most often, primary attention is given to the diagnosis and treatment of diseases in clinical practice, but the possibility of adverse events is neglected, resulting in complications and severe harm to patients. Diarrhea, headache, flatulence, and hyperkalemia are common clinical adverse reactions and therefore were considered the safety endpoints of our assessment as well. Some studies have revealed that veverimer may cause severe gastrointestinal reactions, especially diarrhea (Klaerner et al., 2020). In our study, the veverimer treatment group did not exhibit any significant adverse reactions compared with the control group. 


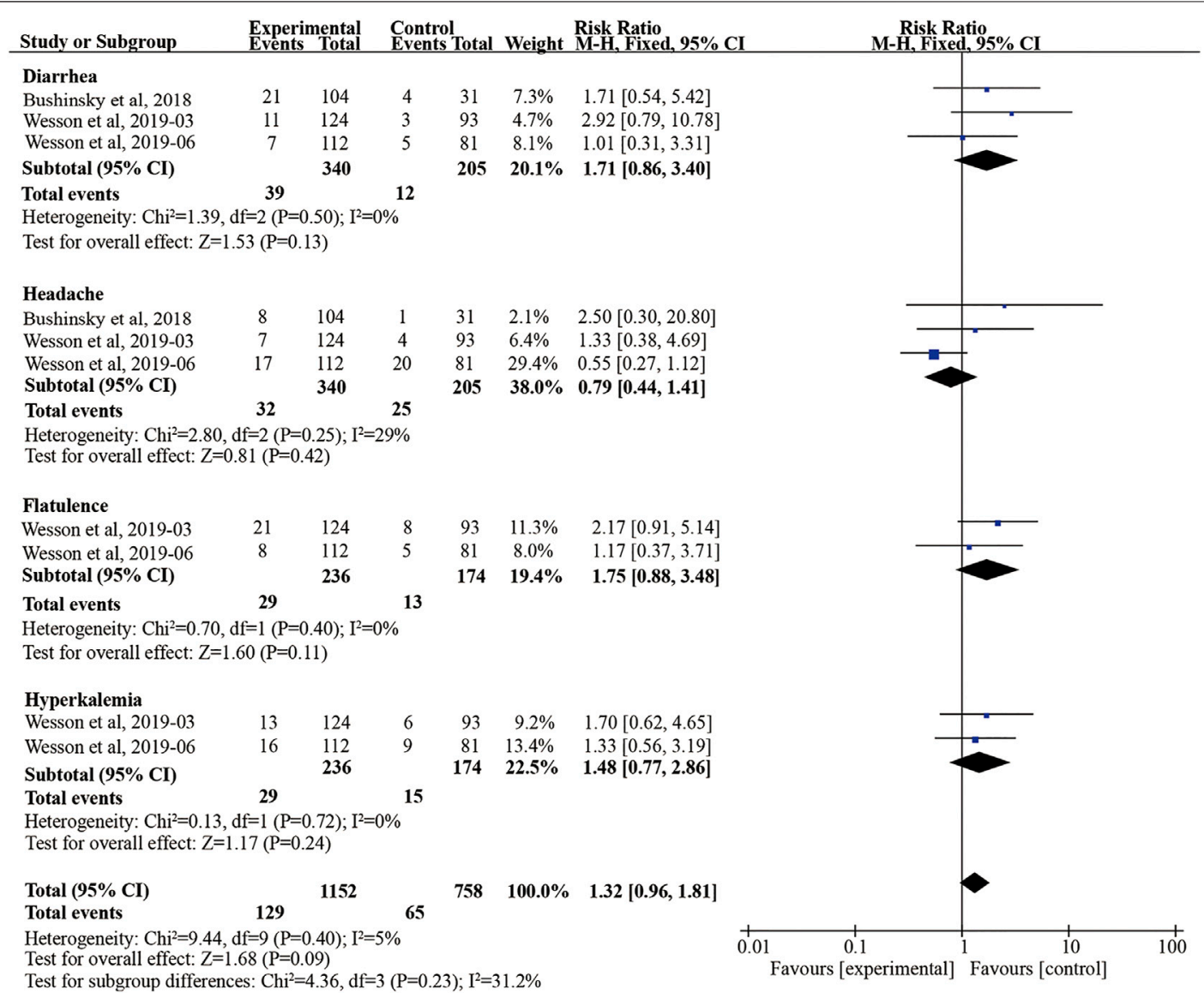

FIGURE 6 | Forest plot for safety outcomes. M-H, Mantel-Haenszel method; 95\% Cl, 95\% confidence interval.

This is the first meta-analysis study regarding veverimer treatment in patients with $\mathrm{CKD}$ and metabolic acidosis to the best of our knowledge. The heterogeneity yielded low for all the included outcomes, indicating a high level of clinical evidence. Nonetheless, it still had shortcomings. First, there was a difference in traits (such as appearance) between the veverimer and placebo groups, and the site staff members could not fully implement the double-blind method. Second, the baseline bicarbonate level and eGFR in the veverimer group were slightly higher than those in the placebo group (Chen and Abramowitz, 2019). However, as a risk element for metabolic acidosis, the eGFR may make it easier for patients in the veverimer group with a slightly higher baseline to achieve the outcome of the evaluation, thereby increasing the heterogeneity of the study. Finally, the trial period of this study was relatively short, and the completed long-term clinical trial was only extended to 52 weeks. Therefore, further studies that could improve on the current data are warranted to evaluate the efficacy and safety of veverimer.

\section{CONCLUSION}

The available clinical evidence indicates that veverimer treatment can be effective and safe in patients with CKD and may correct metabolic acidosis. Nonetheless, multicenter RCTs and large-scale trials are still needed to support this clinical evidence.

\section{DATA AVAILABILITY STATEMENT}

The original contributions presented in the study are included in the article/Supplementary Material, further inquiries can be directed to the corresponding author.

\section{AUTHOR CONTRIBUTIONS}

WL: Search strategy, statistical analysis, manuscript editing; LL: Eligibility criteria; XZ, HD: Extraction of data; ML: Extraction of data, manuscript revision.

\section{FUNDING}

This work was supported by the National Natural Science Foundation of China (Grant No. 81700625). 


\section{REFERENCES}

Abramowitz, M. K., Melamed, M. L., Bauer, C., Raff, A. C., and Hostetter, T. H. (2013). Effects of Oral Sodium Bicarbonate in Patients with CKD. Cjasn 8 (5), 714-720. doi:10.2215/cjn.08340812

Adrogué, H. J., and Madias, N. E. (2020). Veverimer: An Emerging Potential Treatment Option for Managing the Metabolic Acidosis of CKD. Am. J. Kidney Dis. 76 (6), 861-867. doi:10.1053/j.ajkd.2020.07.019

Bailie, G. R., and Massry, S. G. (2005). Clinical Practice Guidelines for Bone Metabolism and Disease in Chronic Kidney Disease: an Overview. Pharmacotherapy 25 (12), 1687-1707. doi:10.1592/phco.2005.25.12.1687

Bushinsky, D. A., Hostetter, T., Klaerner, G., Stasiv, Y., Lockey, C., McNulty, S., et al. (2018). Randomized, Controlled Trial of TRC101 to Increase Serum Bicarbonate in Patients with CKD. Cjasn 13 (1), 26-35. doi:10.2215/CJN. 07300717

Chen, W., and Abramowitz, M. K. (2019). Advances in Management of Chronic Metabolic Acidosis in Chronic Kidney Disease. Curr. Opin. Nephrol. Hypertens. 28 (5), 409-416. doi:10.1097/MNH.0000000000000524

Clement, F. M., Klarenbach, S., Tonelli, M., Johnson, J. A., and Manns, B. J. (2009). The Impact of Selecting a High Hemoglobin Target Level on Health-Related Quality of Life for Patients with Chronic Kidney Disease. Arch. Intern. Med. 169 (12), 1104-1112. doi:10.1001/archinternmed.2009.112

Collister, D., Komenda, P., Hiebert, B., Gunasekara, R., Xu, Y., Eng, F., et al. (2016). The Effect of Erythropoietin-Stimulating Agents on Health-Related Quality of Life in Anemia of Chronic Kidney Disease. Ann. Intern. Med. 164 (7), 472-478. doi:10.7326/m15-1839

Di Iorio, B. R., Bellasi, A., Bellasi, A., Raphael, K. L., Santoro, D., Aucella, F., et al. (2019). Treatment of Metabolic Acidosis with Sodium Bicarbonate Delays Progression of Chronic Kidney Disease: the UBI Study. J. Nephrol. 32 (6), 989-1001. doi:10.1007/s40620-019-00656-5

Dobre, M., Yang, W., Chen, J., Drawz, P., Hamm, L. L., Horwitz, E., et al. (2013). Association of Serum Bicarbonate with Risk of Renal and Cardiovascular Outcomes in CKD: a Report from the Chronic Renal Insufficiency Cohort (CRIC) Study. Am. J. Kidney Dis. 62 (4), 670-678. doi:10.1053/j.ajkd.2013. 01.017

Dobre, M., Yang, W., Pan, Q., Appel, L., Bellovich, K., Chen, J., et al. (2015). Persistent High Serum Bicarbonate and the Risk of Heart Failure in Patients with Chronic Kidney Disease (CKD): A Report from the Chronic Renal Insufficiency Cohort (CRIC) Study. Jaha 4 (4), e001599. doi:10.1161/JAHA. 114.001599

Drawz, P., and Rahman, M. (2015). Chronic Kidney Disease. Ann. Intern. Med. 162 (11), Itc1-16. doi:10.7326/aitc201506020

Goldenstein, L., Driver, T. H., Fried, L. F., Rifkin, D. E., Patel, K. V., Yenchek, R. H., et al. (2014). Serum Bicarbonate Concentrations and Kidney Disease Progression in Community-Living Elders: the Health, Aging, and Body Composition (Health ABC) Study. Am. J. Kidney Dis. 64 (4), 542-549. doi:10.1053/j.ajkd.2014.05.009

Gone, A. R., and Chen, W. (2020). Veverimer for Treatment of Chronic Metabolic Acidosis in Patients with Chronic Kidney Disease. Ann. Transl Med. 8 (22), 1539. doi: $10.21037 / \mathrm{atm}-20-3320$

Heerspink, H. J. L., Holtkamp, F. A., Parving, H.-H., Navis, G. J., Lewis, J. B., Ritz, E., et al. (2012). Moderation of Dietary Sodium Potentiates the Renal and Cardiovascular Protective Effects of Angiotensin Receptor Blockers. Kidney Int. 82 (3), 330-337. doi:10.1038/ki.2012.74

Higgins, J. P. T., Altman, D. G., Gotzsche, P. C., Juni, P., Moher, D., Oxman, A. D., et al. (2011). The Cochrane Collaboration's Tool for Assessing Risk of Bias in Randomised Trials. BMJ 343, d5928. doi:10.1136/bmj.d5928

Hill, N. R., Fatoba, S. T., Oke, J. L., Hirst, J. A., O'Callaghan, C. A., Lasserson, D. S., et al. (2016). Global Prevalence of Chronic Kidney Disease - A Systematic Review and Meta-Analysis. PLoS One 11 (7), e0158765. doi:10.1371/journal. pone. 0158765

Kendrick, J., Shah, P., Andrews, E., You, Z., Nowak, K., Pasch, A., et al. (2018). Effect of Treatment of Metabolic Acidosis on Vascular Endothelial Function in Patients with CKD. Cjasn 13 (10), 1463-1470. doi:10.2215/ cjn. 00380118

Klaerner, G., Shao, J., Biyani, K., Kade, M., Kierstead, P., Gbur, R., et al. (2020). Mechanism of Action of Veverimer: A Novel, Orally Administered,
Nonabsorbed, Counterion-free, Hydrochloric Acid Binder under Development for the Treatment of Metabolic Acidosis in Chronic Kidney Disease. J. Pharmacol. Exp. Ther. 375 (3), 439-450. doi:10.1124/jpet.120.000190 Kraut, J. A., and Madias, N. E. (2016). Metabolic Acidosis of CKD: An Update. Am. J. Kidney Dis. 67 (2), 307-317. doi:10.1053/j.ajkd.2015.08.028

Lomashvili, K., Garg, P., and O'Neill, W. C. (2006). Chemical and Hormonal Determinants of Vascular Calcification In Vitro. Kidney Int. 69 (8), 1464-1470. doi:10.1038/sj.ki.5000297

Menon, V., Tighiouart, H., Vaughn, N. S., Beck, G. J., Kusek, J. W., Collins, A. J., et al. (2010). Serum Bicarbonate and Long-Term Outcomes in CKD. Am. J. Kidney Dis. 56 (5), 907-914. doi:10.1053/j.ajkd.2010.03.023

Moher, D., Liberati, A., Tetzlaff, J., Altman, D. G., and Group, P. (2009). Preferred Reporting Items for Systematic Reviews and Meta-Analyses: the PRISMA Statement. Plos Med. 6 (7), e1000097. doi:10.1371/journal.pmed. 1000097

Navaneethan, S. D., Shao, J., Buysse, J., and Bushinsky, D. A. (2019). Effects of Treatment of Metabolic Acidosis in CKD. Cjasn 14 (7), 1011-1020. doi:10. 2215/CJN.13091118

Peng, S., He, J., Huang, J., Lun, L., Zeng, J., Zeng, S., et al. (2019). Selfmanagement Interventions for Chronic Kidney Disease: a Systematic Review and Meta-Analysis. BMC Nephrol. 20 (1), 142. doi:10.1186/ s12882-019-1309-y

Raphael, K. L. (2019). Metabolic Acidosis in CKD: Core Curriculum 2019. Am. J. Kidney Dis. 74 (2), 263-275. doi:10.1053/j.ajkd.2019.01.036

Raphael, K. L., Wei, G., Baird, B. C., Greene, T., and Beddhu, S. (2011). Higher Serum Bicarbonate Levels within the Normal Range Are Associated with Better Survival and Renal Outcomes in African Americans. Kidney Int. 79 (3), 356-362. doi:10.1038/ki.2010.388

Samsa, G., Edelman, D., Rothman, M. L., Williams, G. R., Lipscomb, J., and Matchar, D. (1999). Determining Clinically Important Differences in Health Status Measures. Pharmacoeconomics 15 (2), 141-155. doi:10.2165/00019053199915020-00003

Shah, S. N., Abramowitz, M., Hostetter, T. H., and Melamed, M. L. (2009). Serum Bicarbonate Levels and the Progression of Kidney Disease: a Cohort Study. Am. J. Kidney Dis. 54 (2), 270-277. doi:10.1053/j.ajkd. 2009.02.014

Susantitaphong, P., Sewaralthahab, K., Balk, E. M., Jaber, B. L., and Madias, N. E. (2012). Short- and Long-Term Effects of Alkali Therapy in Chronic Kidney Disease: a Systematic Review. Am. J. Nephrol. 35 (6), 540-547. doi:10.1159/ 000339329

Wesson, D. E., Mathur, V., Tangri, N., Stasiv, Y., Parsell, D., Li, E., et al. (2019a). Long-term Safety and Efficacy of Veverimer in Patients with Metabolic Acidosis in Chronic Kidney Disease: a Multicentre, Randomised, Blinded, PlaceboControlled, 40-week Extension. The Lancet 394 (10196), 396-406. doi:10.1016/ s0140-6736(19)31388-1

Wesson, D. E., Mathur, V., Tangri, N., Stasiv, Y., Parsell, D., Li, E., et al. (2019b). Veverimer versus Placebo in Patients with Metabolic Acidosis Associated with Chronic Kidney Disease: a Multicentre, Randomised, Double-Blind, Controlled, Phase 3 Trial. The Lancet 393 (10179), 1417-1427. doi:10.1016/ s0140-6736(18)32562-5

Witham, M. D., Band, M. M., Avenell, A., McMurdo, M. E. T., Ogston, S. A., Hampson, G., et al. (2015). Does Oral Sodium Bicarbonate Therapy Improve Function and Quality of Life in Older Patients with Chronic Kidney Disease and Low-Grade Acidosis (The BiCARB Trial)? Study Protocol for a Randomized Controlled Trial. Trials 16, 326. doi:10.1186/s13063-0150843-6

Conflict of Interest: The authors declare that the research was conducted in the absence of any commercial or financial relationships that could be construed as a potential conflict of interest.

Copyright $\odot 2021 \mathrm{Liu}, \mathrm{Li}$, Zhang, Dong and Lu. This is an open-access article distributed under the terms of the Creative Commons Attribution License (CC BY). The use, distribution or reproduction in other forums is permitted, provided the original author(s) and the copyright owner(s) are credited and that the original publication in this journal is cited, in accordance with accepted academic practice. No use, distribution or reproduction is permitted which does not comply with these terms. 